\title{
Spray Refill
}

National Cancer Institute

\section{Source}

National Cancer Institute. Spray Refill. NCI Thesaurus. Code C68939.

A container of medication intended to refill a spray. 